function seems to strengthen another dependent on a similar structure, and also to render it, in some measure, capable of performing the obliterated function. At Norwood, some years ago, I saw a boy, seventeen years of age, who had been born blind, deaf, and dumb. The olfactory, optic, and auditory nerves were equally insensible each to its appropriate stimulus; he could not utter a single articulate sound, and he possessed no voluntary power of locomotion, but possessed a most acute general sensibility on the cutaneous surface, by means of which a single touch made him aware of the presence of a stranger. His sense of taste was good.

In cases of complete division of the spinal marrow below the origin of the phrenics, occasioning a total destruction of sensation and volition in the parts below the section, it is said the reflex actions can be vividly developed, the intensity, in this case, also depending, no doubt, upon the original nervous susceptibility of the subject in a state of integrity of organization. Now when we consider how frequently the filaments from the sympathetic anastomose in the upper portion with cerebral and spinal nerves, and, in the lower portion, with the lumbar and sacral from the spinal marrow, connecting the systems of organic and animal life, and how difficult it is to disconnect them, we find that, although a complete section of the spinal marrow intercepts the direct current of sensation and volition, there is still sufficient indirect connexion with the brain to admit of the transmission of cerebral influence to the muscular system of the extremities for the maintenance of its irritability. Moreover, it is probable, that as the circulation of the blood is continued, in an extremity, by means of anastomosing branches, after the main trunk of the vessel has been tied, so after section, or other division of the spinal marrow, some cerebral influence may be communicated from the upper to the lower segment by means of anastomosis or interlacement of nervous fibrillæ, although not sufficient for the purposes of sensation and volition, which seem to require direct communication.

In the hemiplegic form of paralysis, complete as to the extinction of sensation and volition, I would explain the longcontinued maintenance of the vitality and irritability of the limbs, partly by this collateral or retrograde diffusion of the nervous inftuence, derived from the healthy hemisphere of the cerebral organ. A low degree of vitality increases the irritability of organized structures, a fact often demonstrated by the phenomena of disease. In hemiplegia we see many varieties: it is sometimes partial, at others general; it may be complete or incomplete. Sometimes when the power of motion is completely destroyed, the sensibility is increased to a morbidly acute degree; hence, it does not appear irrational to infer, that if the extinction of the one function is attended with an augmentation of the other, when both are completely destroyed, the limbs may derive direct cerebral influence through the spinal marrow for the support of their vitality, as well as an indirect retrograde supply, by means of the anastomoses of the nervous fibrillie on the cutaneous surface of the body. I cannot here dilate upon the numerous anastomoses of the peripheral extremities of the various nerves in the body, but I think they are sufficiently numerous to convey cerebral influence from the healthy to the diseased side. This subject is, however, one of much intricacy, owing to the varied conditions of paralysis presented to our notice, and requires the collation of multiplied experiments and observations to unravel it. It is also a subject of great interest, and the careful prosecution of it must lead to a better knowledge of the hidden sources of morbid action in the human frame, and to better and more successful modes of treatment.

Oakham, Rutlard, January, 1846.

\section{RUPTURE OF THE UTERUS.}

By Edward C. Tyte, Esq., Harrow.

On the morning of Sunday, the 2nd of Norember, at six o'clock, I was called to attend Mrs. L_- in labour with her third child. The patient was in her twenty-fourth year, healthy, and well-made. I attended her three years ago, when, after a tedious labour, she was delivered of a living child. Upon my arrival, I found she had been in labour since tliree o'clock that morning; that the bowels and bladder had been evacuated freely, and the membranes had ruptured a few minutes before $I$ had entered the room. Upon examining her, I found the os uteri about two-thirds dilated; the head of the child presenting, but very high up; the pelvis well formed; and the external parts dilatable. The pains were tolerably strong, coming on at intervals of five minutes, and gradually increased in intensity, under the influence of which, the os uteri became fully dilated, and the head slowly descended.
About ten o'clock the pains became very severe, recurring with very little intermission, and the head descended low in the pelvis, slightly dilating the perinæum. This state of things continued up to about twelve o'clock, when, after a very strong pain,--not more severe, however, than the preceding ones, $\rightarrow$ complete cessation of nterine action took place, and she complained of severe pain over the lower part of the abdomen. She became desponding, exclaiming that she should die.

From the sudden cessation of the pains, and the occurrence of continued pain over the uterine region, my first impression was, that the uterus was ruptured; but finding there was no hæmorrhage, - that the head of the child had not receded,that she had not felt anything give way internally, and had no vomiting, this idea was abandoned, and I made up my mind to wait, expecting the return of the pains.

At three o'clock P.M., finding the labour pains had not returned, that she still complained of considerable pain, and had vomited twice, I judged it necessary to have a second opinion, and, accordingly, sent for my friend, Mr. Hewlett, of Harrow, who arrived about an hour afterwards with his assistant, Mr. Curtis. We then carefully examined her. The head had receded in some degree, but was in the cavity of the pelvis; there was no impaction, and the rectum and bladder were empty, and we came to the conclusion that the pain she complained of arose from some irregular action of the uterus, excited by the violence of the pains, combined with exhaustion, and that an opiate and a few hours' rest would put a stop to it, and the labour would proceed favourably. An enema, containing forty-five drops of laudanum, was exhibited, and she was undressed and placed in bed. In about a couple of hours the opiate began to take effect; she slept between three and four hours, and awoke at ten o'clock, quite free from pain.

She now took some nourishment, and I expected, from this favourable state of things, that all would go on well. There was, however, no return of labour pains until three o'clock A.M., when slight pains came on, with the effect of advancing the head in a trifling degree; these, however, soon ceased, and her pulse began to flag, and she vomited some gruel she had taken. It now became evident her labour could not be completed without the assistance of art. Upon examining her again, the forefinger could be passed quite round the head, and $\mathrm{Mr}$. Hewlett thought he felt the forehead anteriorly, which afterwards was found to be the case. It was then determined to try and deliver with the vectis, without, however, any success. The forceps were then used, and although there was a slight return of the pains, the head did not advance; finally, the head was perforated with considerable difficulty, and extracted with the craniotomy forceps; the chest and abdomen were then successively eviscerated, and the business completed. The child was of the average size, and, from its appearance, had been dead some little time. Upon applying my hand to the abdomen, I found the uterus firmly contracted, and in a few minutes the placenta was expelled, slightly putrid; there was no hæmorrlage, and the uterus continued contracted. The poor creature bore the operation, which lasted four hours, with great fortitude, appeared comfortable, and partook of some nourishment. She was, however, very much exhausted, and great fears of her well-doing were entertained. I remained with her two hours after her delivery, and shortly after my departure she began to sink, and expired at four o'clock.

Post-mortem examination, twenty-three hours after death.Upon opening the abdomen, there was a considerable escape of air, and there was altogether rather more than a pint of blood in the cavity of the abdomen. Upon examining the uterus, we discovered a transverse rupture, to the extent of upwards of four inches, situated in the anterior part of the cervix near its junction with the vagina; the edges of the ruptured portion were separated, and had a gangrenous appearance; the cavity of the aterus was empty; the walls of the organ were two inches and a half in thickness, and appeared tree from disease. There was intense inflammation of the peritonæum throughout, with some effiusion of lymph, that portion covering the small intestines being most affected. The bladder was empty. The pelvis was measured, and found to be of the natural dimensions, rather capacious than otherwise.

I think there can be no doubt that the rupture of the uterus took place at the cessation of the pains, and was caused by the violent action of the organ, and not by any previous disease of it. Had the ergot been given, this case would have no doubt furnished an illustration of its mischievous effects when improperly administered. The case is an unusual one as none of the common symptoms of rupture of the uterus, if we except the sudden cessation of the labour pains, and the 
occurrence of abdominal pain, were present. The labour had only lasted nine hours, the rupture of the membranes having taken place only six hours previously to the accident. Moreover, there was no impaction, for the head had steadily advanced, and there was no deformity of the pelvis, and there had been no interference with the labour in any way.

Harrow, January, 1846 .

\section{ON SOME ELECTRICAL EFFECTS DEVELOPED} CHIEFLY BY THE GALV ANIC BATTERY.

By George P. T. Hilı, Esq., Filey.

ON sending a current of electricity, by means of the galvanic battery, through fine metallic wires, the most refractory metals are fused with facility, and become incandescent. If thin metallic leaves be employed, they burn with great brilliancy, being dissipated into vapour. Now, on the supposition that the space between the ultimate atoms of a body, independent of the several forces that may be arranged round them, are entirely occupied by heat, $I$ think the evolution of the latter substance, as exemplified in the above cases, may be satisfactorily accounted for. As a consequence of the law, that no two bodies can occupy the same space at the same time, we may suppose that the addition of electricity to a substance causes its heat of combination to be evolved, and thus to become sensible. Otherwise, whence arises this great increase of temperature? The caloric must obviously be supplied through the medium of one or other of the bodies employed; and if we grant this, the inquiry naturally presents itself as to what causes its evolution. Suppose $\mathrm{M}$ to represent a body, and B the heat of combination arranged all around it. Now, if we add to this a portion of electricity, $\mathrm{C}$, on the supposition that this is material, a displacement of part of the specific heat, $B$, must take place, or the body be considerably enlarged. We find that heat is evolved, and must, I conceive, consider the specific heat of the body to have been lessened, for any other source from which this rise of temperature could have been derived is unknown. The form of the body remains unchanged, for, as the specific heat is replaced, atom for atom, or, rather, volume for volume, by the electricity, no condensation can possibly take place. The evolved heat now exerts its action upon the body, which, as in ordinary circumstances, assumes the liquid form, and becomes incandescent.

In these experiments we find quantity of electricity to be the sole requisite. The large battery of Children, though capable of fusing several feet of platinum wire, had an intensity so feeble as not sensibly to cause a divergence of the gold leaves of the electrometer. This is perfectly in accordance with the above theory, for it is clear that the larger the addition of electricity, the greater the diminution of specific heat, whether the tension be high or low. Did the evolved heat proceed from the electric fluid itself, we should of course expect that intensity as well as quantity would be required for the production of these effects. This we know not to be the case. I do not consider the circumstance stated by Dalton, that the specific heats of bodies are greater at high than low temperatures, to be any obstacle to the reception of the above, for, to use an expression of the late Dr. Turner, these phenomena "have been investigated only for matter when in its ordinary state, and probably do not apply in cases of electric excitement. On the other hand, increase of specific heat causes an evolution of electricity. Harris detected electricity, though in exceedingly minute quantity, in the evaporation of distilled water from platinum vessels, when the presence of any chemical action was out of the question. In this we have phenomena directly opposed to the former, but I think they may be considered no more anomalous than the fact, that watery vapour should be decomposed by metallic iron, heated to redness; and that the oxide thus generated should, in its turn, be decomposable by a stream of hydrogen gas. I apprehend, then, that there are other causes in operation, which modify the effects of these most subtle and diffusive bodies, heat and electricity. In the condensation of aqueous vapour, the objects in contact with it likewise show signs of electric excitement. For a portion of vapour contains more specific heat, and less specific electricity, than the same when liquid, and, therefore, before it can assume this form, it must receive electricity from surrounding objects, which thus exhibit signs of its emission.

No heat is evolved when a current of positive or negative electricity only is passed along a body, for in this case the repulsion of the particles confines the fluid to the surface alone, where it cannot influence the internal arrangements of the conductor.

Filey, January, 1846.

\section{THE REPORT OF A CASE OF TETANUS SUCCESS-} FULLY TREATED.

By W. S. Preshaw, Esq., M.R.C.S.Ed., St. Helier's, Jersey. Ox Wednesday, 22nd Oct., 1845, I was requested to visit F. B_-, aged eleven years, residing at George's Town. $\mathrm{He}$ complained of a slight stiffness of the neck; had pain and difficulty on moving his head, legs, \&c. I inquired if he had sustained any injury. He said he had not. Ordered a smart purgative of calomel and jalap, a warm bath for twenty minutes, and an antispasmodic mixture of sulphuric ether and tincture of opium. Towards evening the rigidity of the muscles of the head and neck increased, attended with difficulty in swallowing, tightness about the chest, and inability to move the jaw; the teeth were firmly set, almost every muscle quite rigid, and the spine bent into an arch, leaving no doubt as to the nature of the case. The boy still denied having received any injury. Ordered the spine to be well rubbed with a strong liniment, and the warm bath to be repeated. Next day the boy told me that, on the previous Saturday, (18th Oct.) while stooping at play, a person struck him with a stick over the loins. He did not seem to have felt it much at the time, but the day following said that he felt as if unable to move his legs. Examined the spine, no mark of any injury, but he complained of a slight tenderness over the lumbar region of the spine. Ordered nine ounces of blood to be removed by the cupping-glass from the spot where he seemed to feel most pain. No relief. Next day ordered a dozen leeches to the same place, and a large blister along the spine. The symptoms not relieved. The warm bath as before. Unloaded the lower bowels by a saline enema, and gave an opiate. Next day to administer two grains of calomel every three hours, with a view to affect the mouth; continued for forty-eight hours, and, althongh aided by the copious use of strong mercurial ointment rubbed in inside the thighs, failed in producing the desired effect. Teeches again to the spine. Acted on the bowels by a dose of croton oil, and then administered the oil of turpentine by the mouth, and as an enema. After a due trial this failed. Ordered a blister to the lumbar region of the spine, and two grains of the muriate of morphia to be sprinkled over the blistered surface, and gave the solution in large doses, and at short intervals, but without the least benefit. Several medical friends attended with me, and the above means were persevered in for three weeks. The patient was rapidly sinking from want of nourishment, \&c. A physician who had seen the case, kindly sent me The Lancet, of 22nd March, 1845, in which there is a case recorded by J. W. Stapleton, Esq., "On the Administration of Intoxicating Doses of Atcohol in 'Tetanus;" but the result not bcing favourable, I declined this mode of treatment. I had thought of the Indian hemp, but the conclusions of Dr. Lawrie, of Glasgow, after having tried it in twenty-six cases, gave me little confidence in it.- (London and Edinburgh Monthly Journal of Medical Science, Nov. 1844, p. 946.)

I was, however, inclined to try belladonna, or arnica, when the friends desired a consultation with a French physician. He advised a trial of the hydropathic treatment. Many difficulties presented. The patient was at a distance from my house: I could not receive him there, nor had I liberty to treat him at the public hospital, and the system, to be fairly tried, required more favourable circumstances than those in which the boy was placed. However, I at once enveloped him in a linen sheet, well wrung out of cold water. Over this I placed three or four good blankets, \&c., so as to exclude the air, and prevent evaporation. Kept the patient in this condition for an hour, by which time the temperature of the sheet was $100^{\circ} \mathrm{Fahr}$. The corerings were removed, and the patient plunged into a cold bath, rubbed quite dry, and enveloped in dry blankets for six hours, during which time he perspired very freely, and slept soundly, and said he "felt quite slack:" Repeated the cold bath while in a state of profuse perspiration, and, after an interval of an hour, the wet sheet and subsequent cold bath. This was repeated every six hours, and after twenty-four hours, the jaw relaxed a little, and the spine became less bent. I now placed him under the douche for three minutes, the water falling from a height of twenty feet, and in a stream of one inch and a half diameter. Dried and enveloped in the blankets as before. Able to open the mouth a little. Ordered the sheet and cold bath as before, and ten days from the commencement of the treatment, every symptom yielded, and the boy is now quite well. It cannot be said that "cold water" is a new remedy in such cases, for Dr. Cullen, in his "First Lines of the Practice of Physic," published in 1796 , p. 311 , observes, "The administration of it is sometimes by bathing the person in the sea, or, more 